\title{
Dynamic thiol and disulphide homoeostasis in fibromyalgia
}

\author{
Gulsah Karatas ${ }^{1}$, Ramazan Gunduz ${ }^{1}$, Ismail Haskul², Betul Ustun ${ }^{1}$, Salim Neselioglu \\ Fatih Karatas ${ }^{4}$, Müfit Akyuz ${ }^{1}$, Ozcan Erel ${ }^{3}$
}

\author{
${ }^{1}$ Department of Physical Medicine and Rehabilitation, Karabuk University Education \\ and Research Hospital, Karabuk, Turkey \\ ${ }^{2}$ Department of Biochemistry, Karabuk University Education and Research Hospital, \\ Karabuk, Turkey \\ ${ }^{3}$ Department of Biochemistry, Faculty of Medicine, Yildirim Beyazit University, Ankara, \\ Turkey \\ ${ }^{4}$ Department of Internal Medicine and Medical Oncology, Karabuk University, \\ Education and Research Hospital, Karabuk, Turkey
}

Submitted: 26 November 2018

Accepted: 30 March 2019

Arch Med Sci 2020; 16 (3): 597-602

DOI: https://doi.org/10.5114/aoms.2019.87052

Copyright () 2019 Termedia \& Banach

\section{Abstract}

Introduction: Thiol and disulphide levels are biomarkers that provide useful information about oxidative stress and antioxidant capacity, showing a different homoeostasis in inflammatory and proliferative diseases. We aimed to clarify the possible aetiology of this disease by using thiol and disulphide levels in patients with fibromyalgia, the basis of which has not yet been clearly elucidated.

Material and methods: A total of 156 individuals: 86 patients with fibromyalgia and 70 age-matched controls were included in this prospective non-randomised case-control study. Demographic characteristics including smoking status, body mass index (BMI), the duration of complaints, and pain levels were carefully recorded. Dynamic thiol-disulphide homoeostasis in blood samples was determined by an automatic-spectrophotometric method. The Mann-Whitney $U$ and Student's $t$-test were used to determine the differences between the groups.

Results: Sex, BMI, and smoking status were similar between the groups ( $p=0.62, p=0.09$, and $p=0.64$, respectively). While native thiol levels were found to be high in patients with fibromyalgia $(p=0.018)$, disulphide levels and the rates of disulphide/native thiol and disulphide/total thiol were significantly low ( $p=0.049, p=0.007$, and $p=0.007$, respectively). Correlation analysis showed no significant relationship between thiol-disulphide levels and duration of complaints or pain level.

Conclusions: Thiol-disulphide balance in fibromyalgia was found to be similar to benign proliferative diseases, suggesting that the underlying mechanism is more likely to be of proliferative pattern rather than inflammatory. Additionally, fibromyalgia is not directly associated with increase in oxidative stress. The molecular mechanisms need to be elucidated.

Key words: fibromyalgia, inflammatory, thiol, disulphide, proliferative.

\section{Introduction}

Fibromyalgia is a syndrome characterised by widespread musculoskeletal pain, with an estimated prevalence of $2-8 \%$ in the general population [1]. Patients list a wide range of symptoms and describe
Corresponding author:

Gulsah Karatas

Department of Physical

Medicine and Rehabilitation

Karabuk University

Education and Research

Hospital

1 Alparslan St

78200 Karabuk, Turkey

E-mail: gulsah2206@gmail. com 
the locations of pain along with the presence and severity of fatigue, mood problems, sleep disturbances, headaches, and irritable bowel problems. Also, it is a disease that negatively affects the quality of life, and physical and emotional functions. The first diagnostic criteria for fibromyalgia were published in 1990, defining chronic widespread pain with a number of tender points; however, newer classification criteria for fibromyalgia were proposed in 2010 [2-5]. Various drugs at different doses, such as amitriptyline, pregabalin, gabapentin, and duloxetine, are used in the treatment [6]. The aetiology of the disease is still not clearly understood. Possible aetiological factors include changes in sleep stages as well as hormonal and psychological causes $[7,8]$. According to recent studies, a new possible aetiological cause of fibromyalgia has been found: the changes in the antioxidant system and oxidative stress, which is associated with the aetiopathogenesis of many rheumatic diseases $[9,10]$. The most recently discovered method for showing oxidative stress and antioxidant balance in the human body is thiol-disulphide homoeostasis. While thiols are organosulfur compounds having antioxidant functions with various mechanisms, dynamic disulphide bonds are more likely to be associated with oxidative stress. Dynamic thiol-disulphide homoeostasis is involved in many processes such as protein stabilisation, regulation of enzyme functions, transcription, antioxidant protection, detoxification, cell growth or proliferation, and apoptosis. In proliferative conditions, thiol levels increase and disulphide levels decrease, whereas the opposite of this situation occurs in apoptosis and necrosis. The thiols are oxidised and converted into disulphide bond structures by oxidants in the environment, while the disulphides can be reduced again to thiol structures, so this balance is maintained. Recent studies have reported that dynamic thiol-disulphide homoeostasis reflects the oxidative stress condition and is therefore a novel method for determining the oxidative stress $[11,12]$. Some studies have shown that oxidative stress can also lead to peripheral and central sensitisation and altered nociception [13]. In a previous study including a small number of female patients, it was shown that dynamic thiol-disulphide homoeostasis was affected in fibromyalgia [14]. Given the effects of oxidative stress on central and peripheral sensitisation, evaluating the oxidative stress in patients with fibromyalgia will be helpful in clarifying its aetiology. We therefore aimed to investigate the dynamic thiol-disulphide homoeostasis in a larger patient group (including both female and male patients) in order to demonstrate the importance of oxidative balance in fibromyalgia.

\section{Material and methods}

Patients with fibromyalgia, in whom the diagnosis was made according to the ACR 2010 criteria [4] and who were followed between 2017 and 2018, were included in the study. Age between 18 and 75 years and with fibromyalgia was the inclusion criterion. Exclusion criteria were defined as follows: the presence of any autoimmune disease, history of malignancy, chronic or acute infections, and pregnancy. The control group included healthy subjects who matched the inclusion and exclusion criteria mentioned above. During the patient enrolment, the information about previous medication for fibromyalgia treatment was not available (such as amitriptyline, duloxetine, pregabalin, and gabapentin). Also, it was not noted whether patients had used nonsteroidal anti-inflammatory drugs or received prior physical therapy for any other reason. The demographic characteristics for both groups, such as age, gender, smoking status, body mass index (BMI), and the duration of patients' complaints were carefully recorded. Pain levels were assessed by Visual Analogue Scale (VAS). After $8 \mathrm{~h}$ of fasting, venous blood samples were collected from both groups in the morning and then stored for $20 \mathrm{~min}$ for clotting. The samples were separated by centrifuging for $10 \mathrm{~min}$ at $1500 \mathrm{~g}$. The separated serums were stored in the freezer at $-80^{\circ} \mathrm{C}$ until the time of testing for dynamic thiol-disulphide homoeostasis. Thiol-disulphide homeostasis was measured by automated spectrophotometric method, which was described by Erel and Neselioglu. Measurements were carried out by using a Cobas c501 chemical analyser (Roche Diagnostics, Mannheim, Germany). Briefly, disulphide bonds were first reduced to form free functional thiol groups with sodium borohydride, unused reductant sodium borohydride was consumed and removed with formaldehyde to prevent reduction of DTNB (5, 5'-dithiobis-[2-nitrobenzoic] acid), and all of the thiol groups, including reduced and native, were determined after the reaction with DTNB. Half of the difference between the total thiols and native thiols provided the dynamic disulphide amount. After the determination of native and total thiols, disulphide amounts, disulphide/total thiol percentage ratios $(\mathrm{SS} / \mathrm{SH}+\mathrm{SS})$, disulphide/native thiol percentage ratios (SS/SH), and native thiol/total thiol percentage ratios ( $\mathrm{SH} /$ $\mathrm{SH}+\mathrm{SS})$ were calculated [8]. The serum thiol and disulphide values were shown as $\mu \mathrm{mol} / \mathrm{l}$. The dynamic disulphide homeostasis was determined by the division of the change between total thiol and native thiol. The rates of disulphide/total thiol, disulphide/native thiol, and native thiol/total thiol were calculated according to their percentages. All procedures in this study were in accordance with the 1964 Helsinki declaration and its subsequent amendments or comparable ethical standards. 
Ethics committee approval was obtained from the Ethics Committee of Karabuk University. Written, informed consent was obtained from each participant.

\section{Statistical analysis}

All statistical analyses were carried out using SPSS (v22.0, SPSS Inc., Chicago, IL, USA). The Kolmogorov-Smirnov test and the Shapiro-Wilk test were used to determine whether the data were normally distributed. Standard deviation and mean were used for normally distributed data, and median and range as minimum-maximum values were used for non-normally distributed data. Student's $t$-test was used for the comparison of normally distributed data, and Mann-Whitney $U$ test was used for non-normally distributed data. Spearman and Pearson' tests were used for correlation analysis. The $\chi^{2}$ test was used to determine the difference in terms of smoking status and gender distribution between the groups. $P$-values $<0.05$ were considered as statistically significant.

\section{Results}

A total of 156 subjects were included in the study, including 86 patients with fibromyalgia (12 men, 74 women) and 70 age-matched controls (7 men, 63 women). The median duration of complaints in the fibromyalgia group was 12 (1-240) months and the mean VAS score was $7.09 \pm 1.31$. Gender distribution, BMI, and smoking status were similar between the patient and control groups $(p=0.62, p=0.09$ and $p=0.64$, respectively). In patients with fibromyalgia, native thiol levels were found to be significantly high, while disulphide levels were low ( $p=0.018$ and $p=0.049$, respectively) (Figure 1 ). The patient group had significantly lower disulphide/native

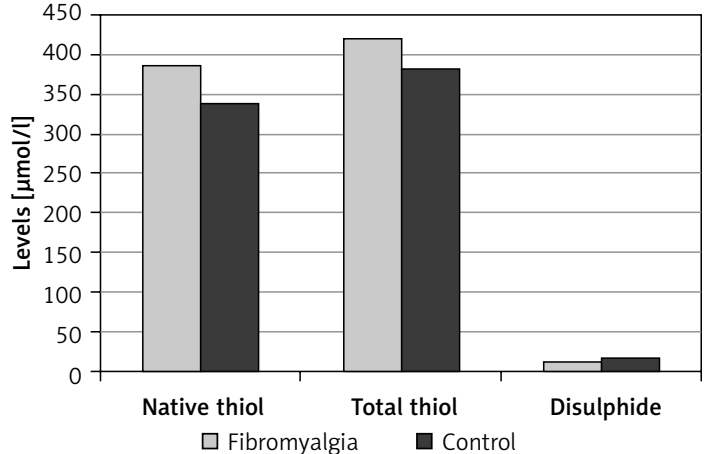

Figure 1. The median levels of native thiol, total thiol, and disulphide in the control and patient groups

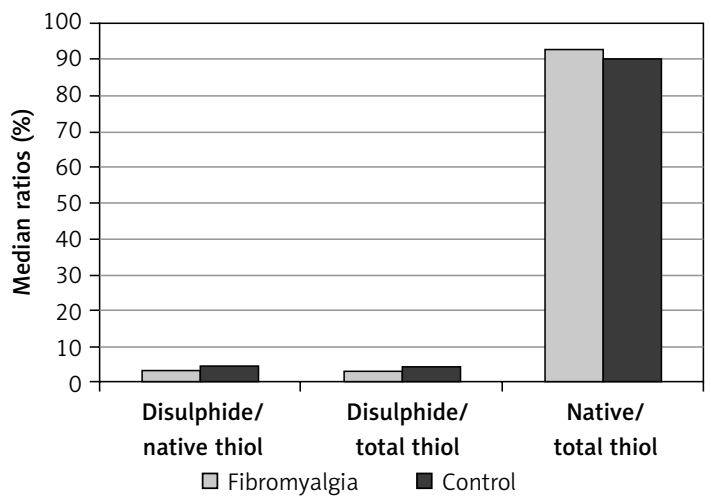

Figure 2. The median ratios of percentages of disulphide to native thiol, disulphide to total thiol, and native to total thiol in the patient and control groups

thiol and disulphide/total thiol rates but higher native thiol/total thiol rate than the control group $(p=0.007, p=0.007$ and $p=0.007$, respectively) (Table I, Figure 2). While correlation analysis revealed no significant correlation between thioldisulphide levels and disease duration or pain level $(p>0.05)$, there was an inverse correlation between age and thiol-disulphide levels as well as between BMI and thiol levels, independent of disease (Table II).

Table I. The comparison of age, BMI, and the rates of disulphide to native thiol, disulphide to total thiol, and native thiol to total thiol between fibromyalgia and control group

\begin{tabular}{|c|c|c|c|}
\hline Variables & Patients with fibromyalgia & Control group & $P$-value \\
\hline Age [years], mean \pm SD & $47.72 \pm 9.52$ & $44.97 \pm 11.61$ & 0.106 \\
\hline $\mathrm{BMI}\left[\mathrm{kg} / \mathrm{m}^{2}\right]$, mean $\pm \mathrm{SD}$ & $29.94 \pm 5.48$ & $28.57 \pm 4.71$ & 0.099 \\
\hline Native thiol $[\mu \mathrm{mol} / \mathrm{l}]$, median (min.-max.) & $387(223-698)$ & $340(211-696)$ & 0.018 \\
\hline Total thiol $[\mu \mathrm{mol} / \mathrm{l}]$, median (min.-max.) & $410(246-750)$ & $383(219-718)$ & 0.060 \\
\hline Disulphide $[\mu \mathrm{mol} / \mathrm{l}]$, median (min.-max.) & $13.6(1.7-36.7)$ & $17.6(3.15-41.09)$ & 0.049 \\
\hline Disulphide/native thiol, median (min.-max.)\# & $3.73(0.27-12.65)$ & $4.71(0.45-13.99)$ & 0.007 \\
\hline Disulphide/total thiol, median (min.-max.) \# & $3.47(0.27-10.09)$ & $4.31(0.45-10.93)$ & 0.007 \\
\hline Native thiol/total thiol, median (min.-max.) & $93(80-99)$ & $90(78-99)$ & 0.007 \\
\hline
\end{tabular}

$B M I$ - body mass index, SD - standard deviation, "the ratios of percentages. 
Table II. The correlation between age, duration, BMI, and VAS and the levels of native thiol, total thiol, disulphide, and the rates of disulphide to native thiol, disulphide to total thiol, and native thiol to total thiol

\begin{tabular}{|lcccccc|}
\hline Variables & Native thiol & Total thiol & Disulphide & $\begin{array}{c}\text { Disulphide/ } \\
\text { native thiol }\end{array}$ & $\begin{array}{c}\text { Disulphide/ } \\
\text { total thiol }\end{array}$ & $\begin{array}{c}\text { Native/ } \\
\text { total thiol }^{\#}\end{array}$ \\
\hline Age & $p=0.001$ & $p<0.001$ & $p=0.012$ & $p=0.44$ & $p=0.45$ & $p=0.44$ \\
& $r=-0.26$ & $r=-0.30$ & $r=-0.20$ & $r=-0.06$ & $r=-0.06$ & $r=0.06$ \\
\hline BMI & $p<0.001$ & $p<0.001$ & $p=0.59$ & $p=0.018$ & $p=0.018$ & $p=0.018$ \\
& $r=-0.034$ & $r=-0.33$ & $r=0.04$ & $r=0.18$ & $r=0.18$ & $r=-0.18$ \\
\hline Duration* & $p=0.52$ & $p=0.59$ & $p=0.59$ & $p=0.89$ & $p=0.89$ & $p=0.88$ \\
& $r=-0.07$ & $r=-0.05$ & $r=-0.05$ & $r=0.01$ & $r=0.01$ & $r=-0.01$ \\
\hline VAS & $p=0.42$ & $p=0.48$ & $p=0.67$ & $p=0.55$ & $p=0.55$ & $p=0.54$ \\
& $r=-0.08$ & $r=-0.07$ & $r=0.04$ & $r=0.06$ & $r=0.06$ & $r=-0.06$ \\
\hline
\end{tabular}

BMI - body mass index, VAS - Visual Analogue Scale, "the ratios of percentages, *duration of complaints (months).

\section{Discussion}

In our study the patient group had significantly higher native thiol levels (antioxidant) and lower disulphide levels (oxidative stress) than healthy controls, with low disulphide/native thiol and disulphide/total thiol rates and high native thiol/ total thiol rate. There was no significant relationship between thiol-disulphide levels and disease duration or pain level. We found that the dynamic thiol-disulphide balance shifted in the direction of thiol, suggesting that dynamic thiol-disulphide homeostasis is associated with an increased shift to the reductive side as a proliferative manner. Similarly, in a study including fewer patients with fibromyalgia, patients were found to have significantly higher native thiol levels, lower disulphide levels, and lower disulphide/total thiol rates than healthy controls. However, in that study, the patient group was composed only of women. Also, smoking status, which is known to have a strong effect on dynamic thiol balance, was not considered in both groups [11]. Unlike in a previous study, our study also included male patients, thus obtaining more accurate data relevant to the general population. Moreover, the number of smokers between the two groups in our analysis was more homogeneous because it is known that smoking would affect these levels [14]. In addition, unlike our findings, in a poster presentation in which the thiol/disulphide haemostasis was investigated in fibromyalgia, the thiol levels were found to be low, whereas the disulphide levels were high; however, because this poster presentation was not turned into a study, the material method part was not clearly understood in detail [15]. In addition, the patients in the fibromyalgia group may be older, have more comorbid disease, and be more intensively smoking than those in the control group. These characteristics, especially cigarette smoking, are the main environmental factors that can reverse this balance [11]. Moreover, Ranzolin et al. reported that oxidative stress was not increased in patients with fibromyalgia
[16]. In another study investigating the oxidative stress status in fibromyalgia through a different marker for oxidative stress, known as "F2 - isoprostanes", oxidative stress was not found to be increased, showing similar findings to our study [17]. On the other hand, some studies have reported that antioxidant levels in fibromyalgia are found to be at low levels. In addition to including different oxidative stress markers and anti-oxidant status, some of these studies have reported that the total thiol levels were similar and low; however, the measurement techniques used in these studies were quite different, with no available measurement for dynamic thiol-disulphide levels [18-20]. Although there are various studies and conflicting results on this issue, the increased thiol and decreased disulphide levels in patients compared to those in the normal population suggest that the thiol cannot be oxidised and converted into disulphide in case of oxidative damage, hence showing that the natural mechanism in these cells is much more different and unique. Similarly to our findings, in a study of depressive patients known to have similar clinical features with fibromyalgia, patients had higher native thiols, lower disulphide levels, and lower disulphide/ thiol rate than those in the control group, while thiol-disulphide levels or balance was not affected by the duration of depression [21]. In addition, in a study on migraine, which has similar clinical features to fibromyalgia, patients with migraine were found to have high level of native thiol and similar level of disulphide compared to a healthy group, with no significant relationship between pain level and these markers [18]. Gumusyayla et al. have stated that high levels of thiol in migraine can be explained by the fact that thiol is a dynamic molecule as well as having antioxidant and pro-oxidant features [22]. While thiol levels were found to be higher in proliferative disease and low-grade gliomas [23], disulphide levels were lower in multiple myeloma, non-small cell lung cancer, prostate cancer, and colon cancer than those in the control group [24-27]. In studies of 
inflammatory diseases, patients had lower thiol levels and higher disulphide levels than those in healthy controls. Likewise, total thiol level was found to be low in some studies including patients with ankylosing spondylitis and juvenile idiopathic arthritis $[28,29]$. The preliminary results of the studies by Erel and Neselioglu showed that plasma disulphide levels were higher in degenerative diseases such as diabetes, bronchiolitis, obesity, and pneumonia, indicating that the balance shifted towards the direction of disulphide in degenerative diseases. Conversely, the plasma thiol-disulphide balance shifted in the direction of thiol in proliferative diseases such as multiple myeloma, colon cancer, bladder cancer, and renal cancer [11].

The major limitations in our study were as follows: current comorbidities and their related medications could not be recorded, and the clinical severity of patients with fibromyalgia was not assessed by long tests (such as the Fibromyalgia Impact Questionnaire and the Beck Depression Inventory). The strength of our work was its large sample size with homogeneous patient group.

In conclusion, patients with fibromyalgia have impairment in thiol-disulphide homoeostasis, with a shift in the direction of thiol, showing similar results to proliferative diseases. Another important finding in our study was that antioxidant levels in the patient group were higher than those in the healthy population, suggesting that fibromyalgia may not be directly associated with an increase in oxidative stress nor with an underlying inflammation or degeneration. The higher thiol levels in our analysis may be due to the insufficient disulphide oxidation of the thiol pool as well as an increased conversion rate of disulphides into thiols. In the coming years and in new studies it would seem to be necessary to focus much more on the common characteristics of fibromyalgia and proliferative diseases. In addition, investigating the reasons of impairment in thiol-disulphide haemostasis in fibromyalgia will be useful fork new treatments. Nevertheless, our findings should be confirmed by prospective studies involving larger numbers of patients.

\section{Acknowledgments}

Many thanks to Suleyman Sahin for his contribution.

\section{Conflict of interest}

The authors declare no conflict of interest.

\section{References}

1. Clauw DJ. Fibromyalgia: a clinical review. JAMA 2014; 311: 1547-55.
2. Wolfe F, Ross K, Anderson J, Russell IJ, Hebert L The prevalence and characteristics of fibromyalgia in the general population. Arthritis Rheum 1995; 38: 19-28.

3. Wolfe F, Smythe HA, Yunus MB, et al. The American College of Rheumatology 1990 criteria for the classification of fibromyalgia. Arthritis Rheum 1990; 33: 160-72.

4. Wolfe F, Clauw DJ, Fitzcharles MA, et al. The American College of Rheumatology preliminary diagnostic criteria for fibromyalgia and measurement of symptom severity. Arthritis Care Res (Hoboken) 2010; 62: 600-10.

5. Roshan R, Shariatpanahi SS, Tavoli A, Qafori Z, Fathi-Ashtiani A. Quality of life in women with fibromyalgia. Arch Med Sci 2009; 5: 267-70.

6. Keshavarz K, Hashemi-Meshkini A, Gharibnaseri Z, Nikfar S, Kebriaeezadeh A, Abdollahi M. A systematic cost-effectiveness analysis of pregabalin in the management of fibromyalgia: an Iranian experience. Arch Med Sci 2013; 9: 961-7.

7. Bellato E, Marini E, Castoldi F, et al. Fibromyalgia syndrome: etiology, pathogenesis, diagnosis, and treatment. Pain Res Treat 2012; 2012: 426130.

8. Bradley LA. Pathophysiology of fibromyalgia. Am J Med 2009; 122: S22-S30.

9. Fatima G, Das SK, Mahdi AA. Some oxidative and antioxidative parameters and their relationship with clinical symptoms in women with fibromyalgia syndrome. Int J Rheum Dis 2017; 20: 39-45.

10. Sánchez-Domínguez B, Bullón P, Román-Malo L, et al. Oxidative stress, mitochondrial dysfunction and, inflammation common events in skin of patients with Fibromyalgia. Mitochondrion 2015; 21: 69-75.

11. Erel O, Neselioglu S. A novel and automated assay for thiol/disulphide homeostasis. Clin Biochem 2014; 47: 326-32.

12. Eroglu S, Haskul i, Aziz V, et al. Dynamic thiol/disulphide homeostasis in patients with uterine myoma. Eur J Obstet Gynecol Reprod Biol 2017; 216: 24-6.

13. Wang ZQ, Porreca F, Cuzzocrea S, et al. A newly identified role for superoxide in inflammatory pain. J Pharmacol Exp Ther 2004; 309: 869-78.

14. Fidan F, Alkan BM, Uğurlu FG, et al. Dynamic thiol/disulphide homeostasis in patients with fibromyalgia. Arch Rheumatol 2017; 32: 112-7.

15. Ekinci B, Bicer C, Baran P, Haliloglu S, Uzkeser H, Carlioglu A. How does thiol/disulphide homeostasis change in fibromyalgia patients? Arthritis Rheum 2016; 68 (Suppl 10): abstract 38.

16. Ranzolin A, Duarte AL, Bredemeier M, et al. Evaluation of cytokines, oxidative stress markers and brainderived neurotrophic factor in patients with fibromyalgia - a controlled cross-sectional study. Cytokine 2016; 8: 25-8.

17. Chung CP, Titova D, Oeser A, et al. Oxidative stress in fibromyalgia and its relationship to symptoms. Clin Rheumatol 2009; 28: 435-8.

18. Altindag O, Gur A, Calgan N, Soran N, Celik H, Selek S. Paraoxonase and arylesterase activities in fibromyalgia. Redox Rep 2007; 12: 134-8.

19. Sarıfakıoğlu B, Güzelant AY, Güzel EÇ, Güzel S, Kızıler AR. Effects of 12-week combined exercise therapy on oxidative stress in female fibromyalgia patients. Rheum Int 2014; 34: 1361-7.

20. Cordero MD. Oxidative stress in fibromyalgia: pathophysiology and clinical implications. Reumatol Clin 2011; 7: 281-3.

21. Dirican N, Dirican A, Sen O, et al. Thiol/disulfide homeostasis: a prognostic biomarker for patients with 
advanced non-small cell lung cancer? Redox Rep 2016; 21: 197-203.

22. Gumusyayla S, Vural G, Bektas H, Neselioglu S, Deniz O, Erel O. A novel oxidative stress marker in migraine patients: dynamic thiol-disulphide homeostasis. Neurol Sci 2016; 37: 1311-7.

23. Inal BB, Emre HO, Baran O, et al. Dynamic thiol-disulphide homeostasis in low-grade gliomas: preliminary results in serum. Clin Neurol Neurosurg 2017; 161: 17-21.

24. Dirican N, Dirican A, Sen O, et al. Thiol/disulfide homeostasis: a prognostic biomarker for patients with advanced non-small cell lung cancer? Redox Rep 2016; 21: 197-203.

25. Hanikoglu F, Hanikoglu A, Kucuksayan E, et al. Dynamic thiol/disulphide homeostasis before and after radical prostatectomy in patients with prostate cancer. Free Radic Res 2016; 50 (Suppl 1): S79-S84.

26. Guney T, Kanat IF, Alkan A, et al. Assessment of serum thiol/disulfide homeostasis in multiple myeloma patients by a new method. Redox Rep 2017; 22: 246-51.

27. Jonas CR, Ziegler TR, Gu LH, Jones DP. Extracellular thiol/ disulfide redox state affects proliferation rate in a human colon carcinoma (Caco2) cell line. Free Radic Biol Med 2002; 33: 1499-506

28. Dogru A, Balkarli A, Cetin GY, et al. Thiol/disulfide homeostasis in patients with ankylosing spondylitis. Bosn J Basic Med Sci 2016; 16: 187-92.

29. Altinel Acoglu E, Erel O, Yazilitas F, et al. Changes in thiol/disulfide homeostasis in juvenile idiopathic arthritis. Pediatr Int 2018; 60: 593-6. 